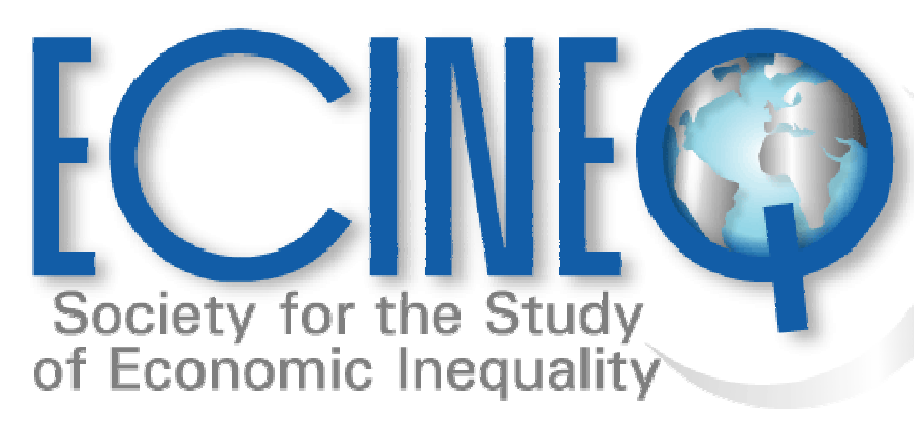

\author{
Working Paper Series
}

Modeling the evolution of age-dependent Gini coefficient for personal incomes in the U.S. between 1967 and 2005

Ivan O. Kitov 


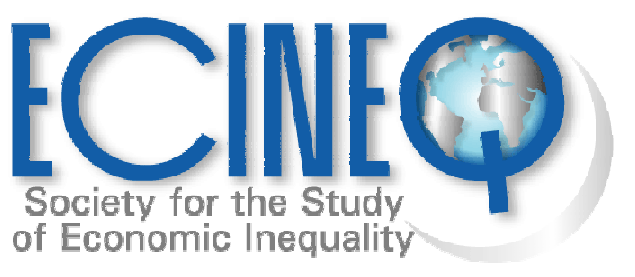

ECINEQ 2008-95

September 2008

www.ecineq.org

In memory of Professor V.N.Rodionov

\title{
Modeling the evolution of age-dependent Gini coefficient for personal incomes in the U.S. between 1967 and 2005
}

\author{
Ivan O. Kitov * \\ Russian Academy of Science
}

\begin{abstract}
This study validates the microeconomic model defining the evolution of personal incomes in the U.S. Because of a large portion of population not reporting any income, any comprehensive modeling of the overall personal income distribution (PID) is complicated. Age-dependent PIDs allow overcoming this shortcoming since the portion of population without income is very low $(<4 \%)$ for ages over 45 years. It is demonstrated that the evolution of Gini coefficient, for the years with a good PID resolution, can be accurately $(<0.005)$ predicted.

As the overall PIDs, the empirical age-dependent (density) PIDs collapse to practically one curve when normalized to cumulative growth in personal income and total population in given age groups for the period between 1967 and 2005. This allows exact prediction of Gini coefficient and other measures of inequality, which are defined by PID. Therefore, these measures of income inequality are only of secondary importance.

In all age groups, the model predicts slightly decreasing Gini coefficients between 1977 and 2005. The overall $G$ is approximately constant, however. The Pareto law index, $k$, undergoes significant changes over age: increases from the youngest age to approximately 67 years of age, and then drops. This index defines the roll-off at the highest incomes.
\end{abstract}

Key words: personal income distribution, age, mean income, microeconomic modelling, USA, real GDP, macroeconomics

JEL Classification: D01, D31, E17, J1, O12

*Address of correspondence: ikitov@ mail.ru 